\title{
VEGFR1-1084 Peptide Vaccine
}

National Cancer Institute

\section{Source}

National Cancer Institute. VEGFR1-1084 Peptide Vaccine. NCI Thesaurus. Code C77895.

A peptide vaccine containing an HLA-A $* 2402$-restricted epitope of vascular endothelial growth factor receptor 1 (VEGFR1 or Flt-1) with potential immunostimulating, antiangiogenic, and antineoplastic activities. Upon vaccination, VEGFR1-1084 peptide vaccine may stimulate a cytotoxic T lymphocyte $(C T L)$ response against VEGFR1expressing endothelial cells of the tumor microvasculature, which may inhibit tumor angiogenesis and tumor cell proliferation. VEGFR1, a receptor tyrosine kinase, may be overexpressed on endothelial cells of the tumor microvasculature and is associated with tumor cell proliferation, invasion and tumor ang iogenesis. HLA-A*2402 is an MHC class I molecule that presents antigenic peptides to CD8+ T cells; epitope design restricted to epitopes that bind most efficiently to HLA-A*2402 may improve antigenicity. 